\title{
Analysis of Effect of Change Source to Surface Distance (SSD) and the Field Size to Distribution Dose Using Monte Carlo Method-EGSnrc
}

\author{
Intan Dillia Nurhadi ${ }^{*}$, Ridwan Ramdani ${ }^{1}$, Freddy Haryanto ${ }^{2}$, Yudha Satya Perkasa ${ }^{1}$, Mada \\ Sanjaya ${ }^{1}$ \\ ${ }^{1}$ Physics Laboratory Modeling Faculty of Sains and Tecnology, State Islamic University Sunan Gunung Djati, St.A.H. \\ Nasution No.105, Bandung 40614, Indonesia \\ ${ }^{2}$ Physics Laboratory Nuclear Faculty of Math and Science Institute of Technology, S.t Ganesha No.10, Bandung \\ 40132, Indonesia
}

( Received: November 18, 2016, Revised: February 10, 2017, Accepted: January 13, 2019 )

\begin{abstract}
Research was conducted to analyze the effect of changes in the distance radiation source to the surface it is called the medium Source to Surface Distance (SSD) and wide exposure field (Field Size) on the distribution of the dose in linear air Accelerator (LINAC) using Monte Carlo - EGSnrc. Monte Carlo simulation is used for modeling and simulation head linac at BEAMnrc. Virtual model of the linac is made based on the data characteristics of the aircraft Linac Varian Clinac $\mathrm{iX}$ then its output obtained information characteristic photon beam using BEAMDP, while modeling and simulation phantom done on DOSXYZnrc with the size of the phantom is $(40 \times 40 \times 40) \mathrm{cm}^{3}$, with the material in the form of water, using a beam of photons $6 \mathrm{MV}$, testing variation $\mathrm{SSD}$ at $80 \mathrm{~cm}, 90 \mathrm{~cm}, 100.1 \mathrm{~cm}, 110 \mathrm{~cm}, 120 \mathrm{~cm}$ and variation field size is $(6 \times 6) \mathrm{cm}^{2},(10 \times 10) \mathrm{cm}^{2},(20 \times 20) \mathrm{cm}^{2}$ to obtain disribution of dosage form of curves Percentage Depth dose (PDD) and Profile dose. The results showed that the smaller distance radiation source to the surface of the medium (SSD) and the greater the broad field (field size), then increasing the dose distribution is obtained. In the SSD and Field Size variation, the radiation dose will continue to rise significantly from the surface of the medium $0 \mathrm{~cm}$ to a depth of maximum dose (Dmax), then the dose began to decline after passing the depth Dmax.
\end{abstract}

Keywords: Monte Carlo - EGSnrc, LINAC, SSD, field size, the photon beam 6mV, PDD, and profiles dose

\section{INTRODUCTION}

Purpose of radiotherapy is to seek cancer cells get a dose as possible and try to get minimal radiation exposure to healthy tissue surrounding [1]. Linear Accelerator (LINAC) is an aircraft that uses microwaves to accelerate charged particles, such as high-energy electrons through a linear tube that is capable of changing the electron kinetic energy. Table PDD for clinical use is usually measured on a standard SSD (80 or $100 \mathrm{~cm}$ ) [2]. However, in certain situations SSD can be rearranged in different patients because of the standard SSD used. For example, required a larger SSD or smaller for treatment technique that involves field smaller or larger, than that available on standard SSD. Field

\footnotetext{
* Corresponding author

E-mail address: intandillia_nurhadi@yahoo.com
}

size is the size of the field or the magnitude of the field widely used in radiation. Secondary collimators often called jaws,namely a device in the linac is used to measure the radiation field that will be used in the planning of radiation [2].

\section{THEORY/CALCULATION}

Monte Carlo simulation is a method that describes the data retrieval random and its probabilistic opportunities. Monte Carlo simulations follows the eventful trip particles ranging from particles of life when released from its source, how much distance the particles then various interactions experienced by the particle when it penetrates the material, and the interaction of what happen along the way to the extent desired, namely until the particles die 
(because loose, absorbed,.etc) [3]. EGSnrc is a program to simulate the transport of photons and electron beam algorithms using Monte Carlo method [4]. EGSnrc structure can be seen in the picture below,

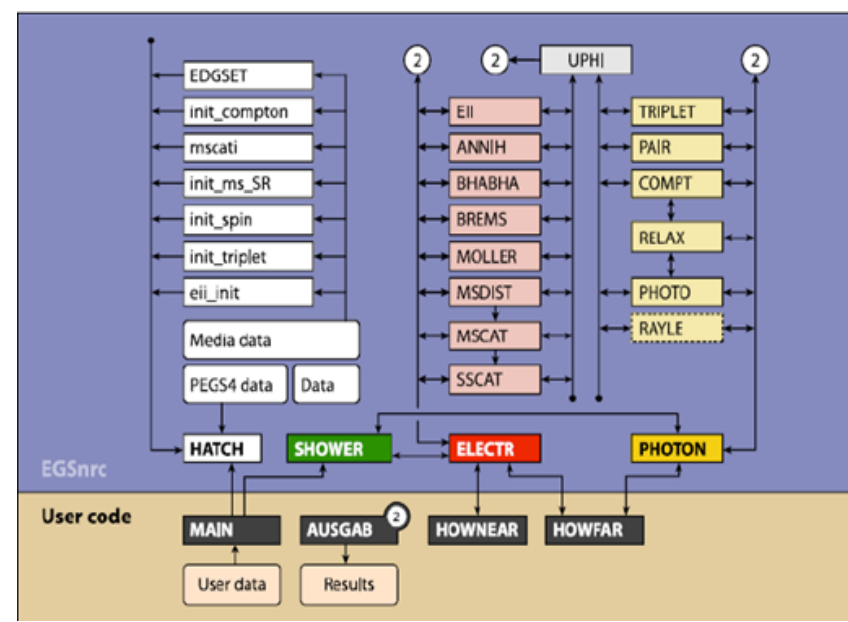

Figure 1. Code EGSnrc program consists of user code and EGSnrc code [4].

EGSnrc has two code system consisting of user code and egscode.In. User MAIN code is the main program it is the place to initialize simulations to determine the characteristics of photons in the form of photons initial position (source), the type of material, type of medium, transport radiation (electrons or photons) to the initial energy.

There subroutine AUSGAB whose function is to record all the desired parameters, subroutine howfar and HOWNEAR on user code eachhas a function to adjust the step size with photons or electrons. EGSnrc using Monte Carlo calculation to vote in the tranport particle radiation, determine the interaction of what happens when a photon strikes a material. EGSnrc has two usercode,including BEAMnrc and DOSXYZnrc [5].

\section{Transfor Particle In The Simulation Monte Carlo}

Numbers random and probability distributions are used to simulate any traces of the particle trajectories of modeling Monte Carlo in the course of radiation, selection of the type of interaction at every step journey and the determination of the state of the particle (energy and direction) was performed using the two elements. Monte Carlo simulations in the course of radiation can be divided into four main steps:

1 Selection of the distance to the next interaction.

2 Journey to the point of interaction to take geometry in.
3 Selection of the type of interaction.

4 Simulation interactions are selected.

These step are repeated until the particles out leaving the simulation geometry or if a low energy below the energy specification, which is the energy of particles are assu to stop and absorbed in the medium [3].

\section{Travel Simulation Photon}

Simulation of traveling photon done in 3 steps where the photons have a position, direction, and energy. In one step, the photons cover the distance at random. The first step determines the distance that the photon x.Probability mileage up to experience the interaction of photons are given by the exponential attenuation law. The equation is represented by the Monte Carlo Probability Distribution Functions (PDF),

$$
f(x)=\sigma_{T}(E) e^{-\sigma_{T}(E) x}
$$

which $\sigma_{T}(E)$ is a cross section of the total. The distance traveled photons $(x)$ obtained by integrating the PDF to obtain Cumulative Distribution Function (CDF),

$$
f(x)=\int_{0}^{x} \sigma_{T}(E) e^{-\sigma_{T}(E) x} d x=1-e^{-\sigma_{T}(E) x}
$$

$f(x)$ normalized $f(\sim)=1$ so, then the inverse CDF,

$$
x=-\frac{1}{\sigma_{T}(E)} \ln (1-f(x))
$$

random numbers $\xi$ replace $f(x)$ with a value of 0 to 1 .

$$
x=-\frac{1}{\sigma_{T}(E)} \ln (1-\xi)
$$

Values random numbers $\xi$ the distributed with alues from 0 to 1 value $(1-\xi)$ is also worth from 0 to 1 , the computing time will be reduced by using the following equation,

$$
x=-\frac{1}{\sigma_{T}(E)} \ln \xi
$$

equation (5) is used to simulate the mileage to experience the next interaction. The second step is the selection of the type of interaction. Types of interaction selected randomly, such as the photoelectric effect, compton scattering, pair production, and rayleigh scattering. The method is 
called rejection, that is by looking for new PDF value by dividing the maximum value of PDF to PDF,

$$
P D F^{\prime}=\frac{P D F}{P D F_{\max }}
$$

maximum value $P D F$ the is 1 . In this step $P D F$ is maximum $\sigma_{T}(E)$ that thecross section of the total, or can be written $\sum_{j=1}^{n} \sigma_{j}$ while $f(x)$ the probability of interaction types. So it can be written equation,

$$
f(i)=\frac{\sum_{j=1}^{i} \sigma_{j}}{\sum_{j=1}^{n} \sigma_{j}}, n \geq i
$$

where $n=$ the total amount from the interaction (eg, $n=4$, for 4 the possibility of interaction, the photoelectric effect, compton scattering, pair production, scattering reyleigh). $i=$ integer to show the interaction numbers $\left(\sigma_{1}\right.$ cross section of thefor photoelectric, $\sigma_{2}$ for compton, $\sigma_{3}$ for the production of the couple, and $\sigma_{4}$ for rayleigh). Furthermore, to determine the type of interaction that is the random number.

$$
f(i-1)<\xi<f(i)
$$

Suppose numbers random taken have value between $f(2)$ and $f(3)$, the photoelectric effect is rejected ,then compton effect was also rejected, and for the production of new couples welcome. Thus, the interaction that occurs is the production partner. The third step is the selection of a random corner of the particles and the new energy of the photons possessed. The probability is influenced by the initial energy photons and medium traversed.

Possiblity of new particles will be obtained according to the results of each interaction, the position, direction and energy for new particles will be stored in the stack [3].

\section{EXPERIMENTAL METHOD}

Modelling linac head is divided into two parts, namely the head linac part 1 (dependent patient component) and head linac part 2 (independent patient component), these are done to reduce the simulation time needed when varying the field size and SSD. The dose that simulated by DOSXYZnrc the direction of the $\mathrm{z}$-axis is known as the Percentage Depth Dose (PDD) and the dose in the horizontal direction (dose profile) y-axis. The components contained in the linac Varian Clinac iX High energy $6 \mathrm{MV}$ x-rays including the target, the primary collimator, Flatteing filter, monitor chamber, the jaws (secondary collimator) and Multileaf Collimator (MLC).

Source of photons generated from the interaction of high-energy electrons are imposed on the target(tungsten) to produce $\mathrm{X}$-rays. The size and number voxel used in the phantom PDD and dose

\begin{tabular}{|c|c|c|c|}
\hline Wick & Total Voxel & Size Voxel $(\mathrm{cm}$ & \\
\hline \multirow{3}{*}{$\mathrm{X}$} & \multirow{3}{*}{3} & Voxel -1 & 18.5 \\
\hline & & Voxel -2 & 3 \\
\hline & & Voxel -3 & 18.5 \\
\hline \multirow{3}{*}{$\mathrm{Y}$} & \multirow{3}{*}{3} & Voxel -1 & 18.5 \\
\hline & & Voxel -2 & 3 \\
\hline & & Voxel -3 & 18.5 \\
\hline \multirow{3}{*}{$\mathrm{Z}$} & \multirow{3}{*}{36} & Group -1 (2 Voxel) & 0.4 \\
\hline & & Group -2 (16 Voxel) & 0.2 \\
\hline & & Group -3 (18 Voxel) & 2 \\
\hline
\end{tabular}
profiles are shown in the Table below.

Table 1. The size and number voxel used in the phantom PDD.

Table 2. The size and number of voxels used for phantom dose profile

\begin{tabular}{cccc}
\hline Wick & Total Voxel & Size Voxel $(\mathrm{cm})$ \\
& & Voxel -1 & 19 \\
$\mathrm{X}$ & 3 & Voxel -2 & 2 \\
& & Voxel -3 & 19 \\
\hline \multirow{3}{*}{$\mathrm{Y}$} & \multirow{3}{*}{42} & Group -1 (7 voxel) & 2 \\
& & Group -2 (10 voxel) & 0.2 \\
& & Group -3 (8 voxel) & 1 \\
& & Group -4 (10 voxel) & 0.2 \\
& & Group -5 (7 voxel) & 2 \\
\hline \multirow{2}{*}{ Z } & 3 & Voxel -1 & 9.5 \\
& & Voxel -2 & 1 \\
& & Voxel -3 & 29.5
\end{tabular}




\section{RESULTS AND DISCUSSION}

(a)
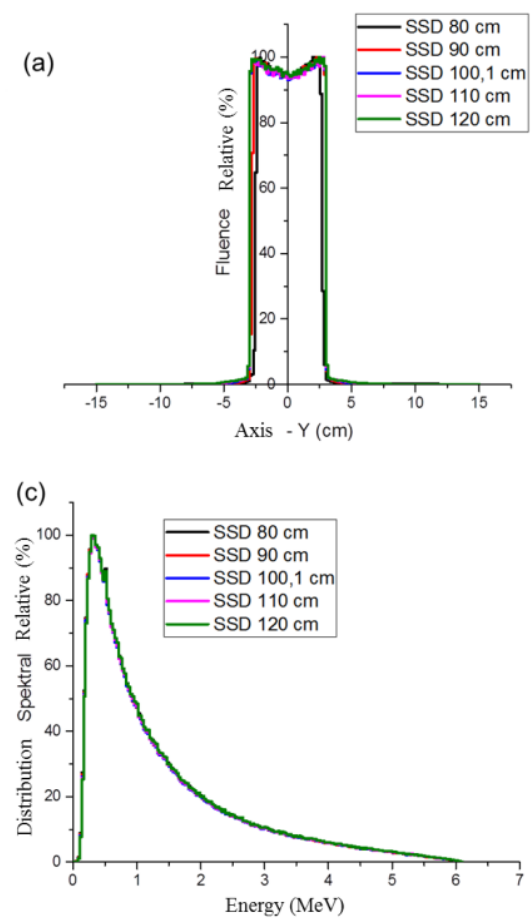
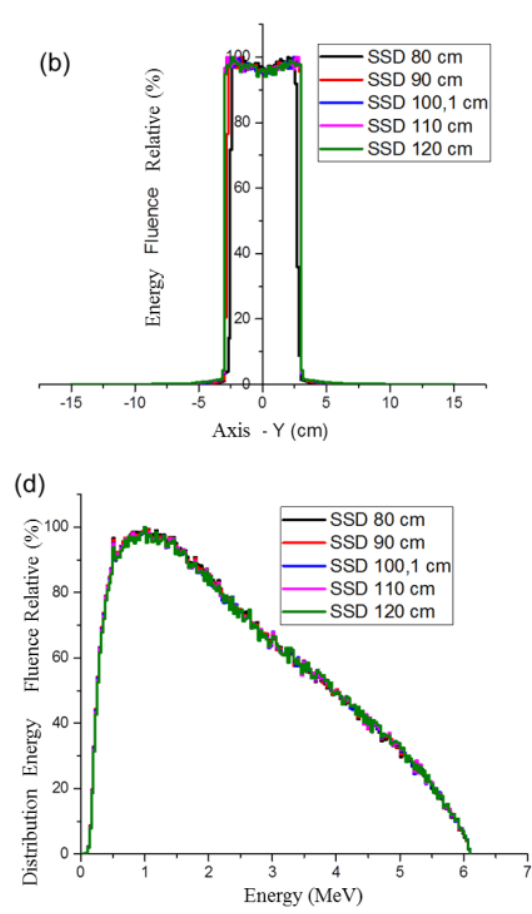

Figure 2. Characteristics of the photon beam with a field size $(6 \times 6) \mathrm{cm}^{2}$

(a)

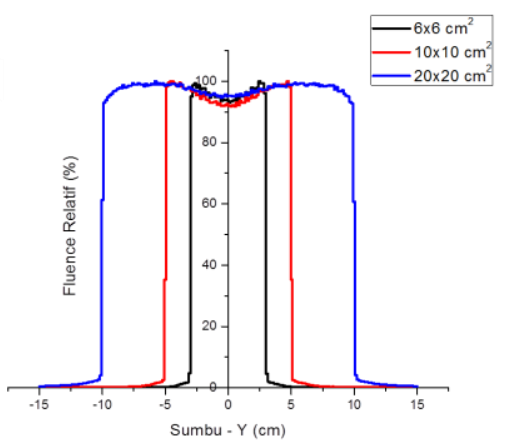

(c)

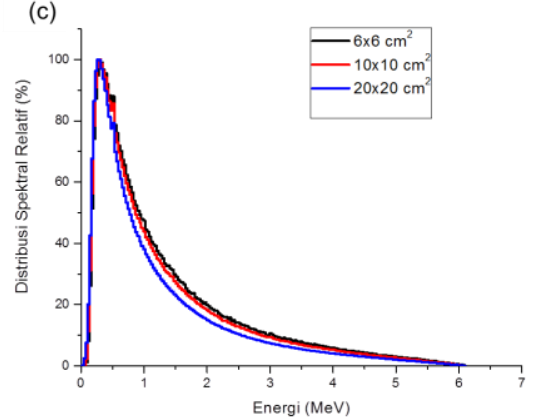

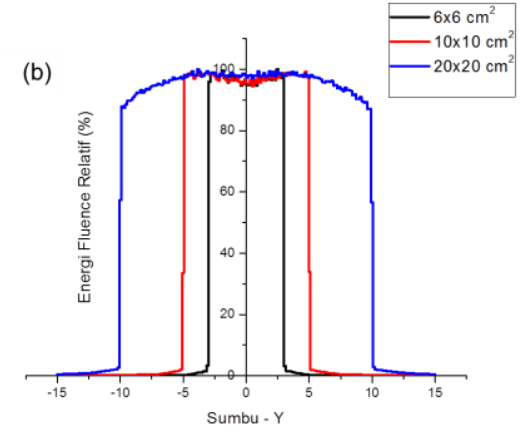

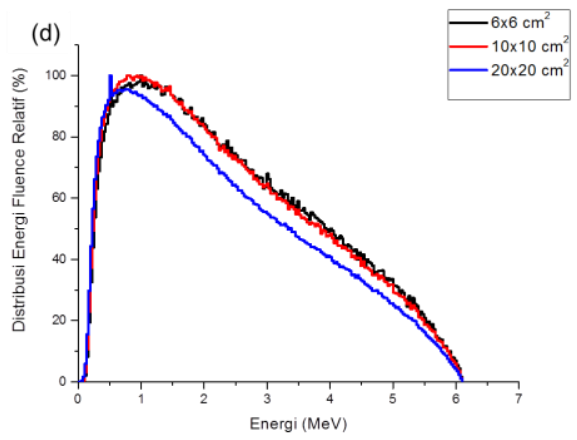

Figure 3. Characteristics of the photon beam with the energy of $6.1 \mathrm{MeV}$ electron early, SSD $100.1 \mathrm{~cm}$ on the surface of the phantom,to variations in field size $(6 \times 6) \mathrm{cm}^{2},(10 \times 10) \mathrm{cm}^{2}$, and $(20 \times 20) \mathrm{cm}^{2}$, (a) Fluence relative to fluence total in the $\mathrm{Y}$-axis, (b) Energy fluence relative to the energy fluence total-axis direction Y, (c) Distribution of spectral relative to the spectral distribution of the total, and (d) Distribution of energy fluence relative to the energy total fluence. 
After that the variations $S S D 80 \mathrm{~cm}, 90 \mathrm{~cm}$, $100.1 \mathrm{~cm}, 110 \mathrm{~cm}$ and $120 \mathrm{~cm}$ on the surface of the phantom;(a) Fluence relative to fluence total in the Y-axis, (b) Energy fluence relative to the energy fluence total-axis direction $\mathrm{Y}$, (c) Distribution of spectral relative to the spectral distribution of the total, and (d) Distribution of energy fluence relative to the energy fluence total.

In each graph, use the number of bins ( $\mathrm{N}$ bin) by 200 in the plot for the Y-axis direction. Figure (a) states fluence relative to the position of the Yaxis, which shows the number of particles in the simulation unity of matter that is the cross sectional area of the crushed material particles. The Particle in the central regions more conentrate due to the sources used are iSource - 19, which is a gaussian distribution where particles accumulate at the center, on a variety of SSD seen that SSDs are getting bigger or smaller does not have a major impact to the change chart fluence on the position in the axis $\mathrm{Y}$ due to changes very little. While on the variation field size seen that the field size is larger has fluence a larger and higher due to the contribution from scattering effect is greater with increasing fieldsize.(b) stating the number of particles is multiplied by the kinetic energy of each particle stored in the bin position -Y axis direction, it appears that the SSD variation greater or less do not have a major impact in the graph changes the energy fluence of the $\mathrm{Y}$-axis position because the changes are very small. While variations in field size is high and energy fluence greater because the photons have the greatest contribution in the formation of energy fluence, the photon beam this phenomenon in accordance with the formula fraction of the electron energy is converted into $\mathrm{X}$ - ray bremsstrahlung.(c) declare the spectral distribution of each particle, for a variety of SSD is the curve of symmetry in all SSD. As for variations in fieldsize, shows that the curve is not symmetrical with an average energy of about $0.5 \mathrm{MeV}$. Dose distribution chart shows the number of dose distributions in the amount of radiation energy, dose distributions decreased with increasing energy. (d) shown in varisai SSD, the distribution of energy fluence seen constant and there are no significant differences, it is stated the distribution of energy fluence that normalization against widtth bin and the number of initial particles are used. While the variation of field size greater it is mean that the energy distribution curve fluence the lower/ smaller.energy distribution graph Fluence indicates the number of doses distributed to a number of radiant energy.

\section{Results Determination of Percentage Depth Dose (PDD) and Profile Dose-Varying Source to Surface Distance (SSD)}

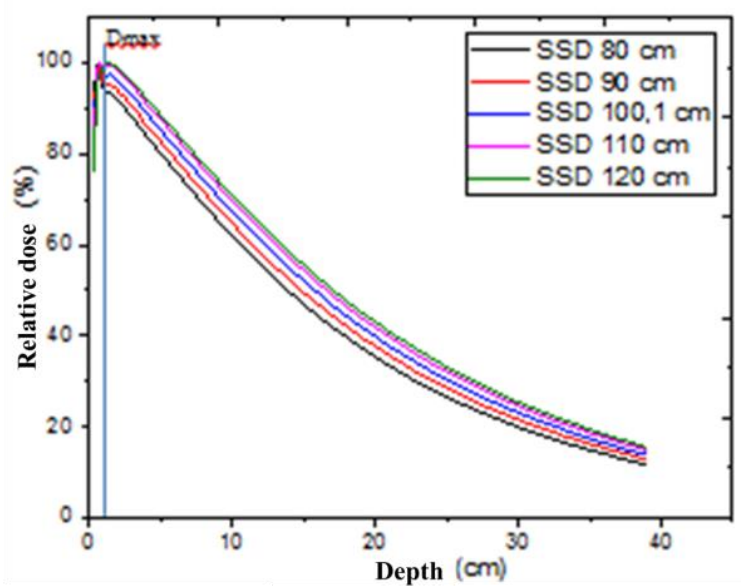

(a)

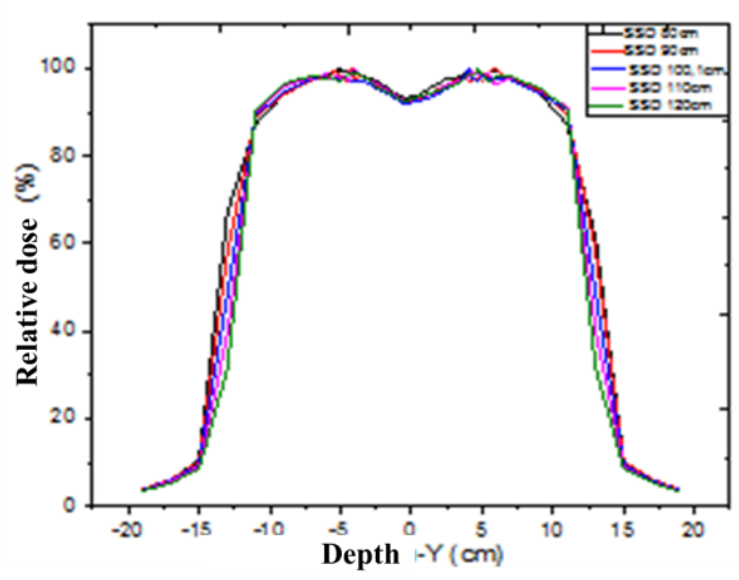

(b)

Figure 4. Variation SSD with a field size $(20 \times 20) \mathrm{cm}^{2}$ at the beginning of $6.1 \mathrm{MeV}$ electron energy. (A) PDD graph shows the depth dose distributions (b) The graph shows the dose profile dose distributions to the $\mathrm{Y}$-axis field.

Dmax with a dose of $100 \%$ less likely to experience changes in the SSD $80 \mathrm{~cm}, 90 \mathrm{~cm}, 100.1$ $\mathrm{cm}$ at a depth of $0.6 \mathrm{~cm}$ and changes in the SSD 120 $\mathrm{cm}$ with a depth of $1.3 \mathrm{~cm}$. When the distance is smaller SSD will affect the increased value of the dose in the build-up due to the contribution of electrons results of the photoelectric effect and the scattering of radiation is dominant. It is caused by the electron has a range shorter than the photon as experienced interaction with the constituent particles of medium phantom and a dose in the region fall off the dose deposited in the medium phantom water will decrease after passing a dose depth maximum or Dmax,due to reduced interaction of photons with a medium, if the photon energy is greater, then the energy is transferred to electrons also getting bigger, 
so the range of secondary electron reaction will further or deeper.

SSDs are getting bigger, there are no significant changes due to the varying distances of SSD is not too far away. The symmetry of the dose profile curve is very important because of the dose profile curve can be seen from the symmetry of the mean photon beam dose homogeneously dispersed in the target area.

\section{Results Determination of Percentage Depth Dose (PDD) and Profile Dose-Varying Field Size}

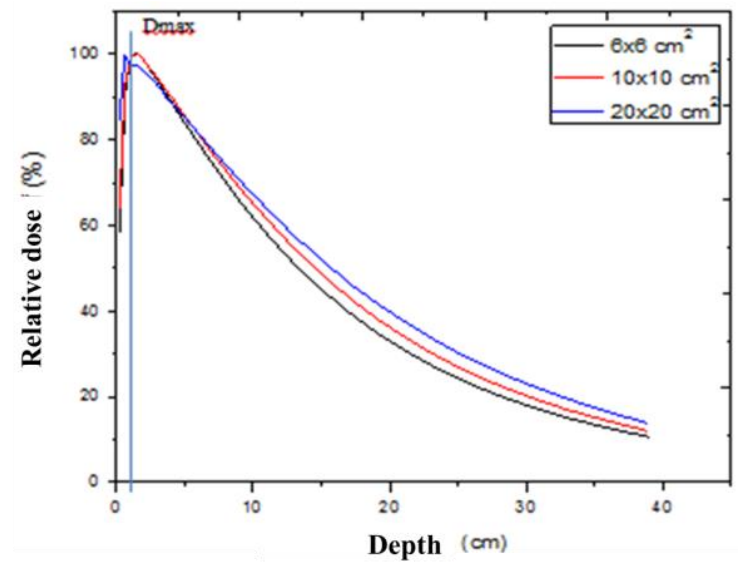

(a)

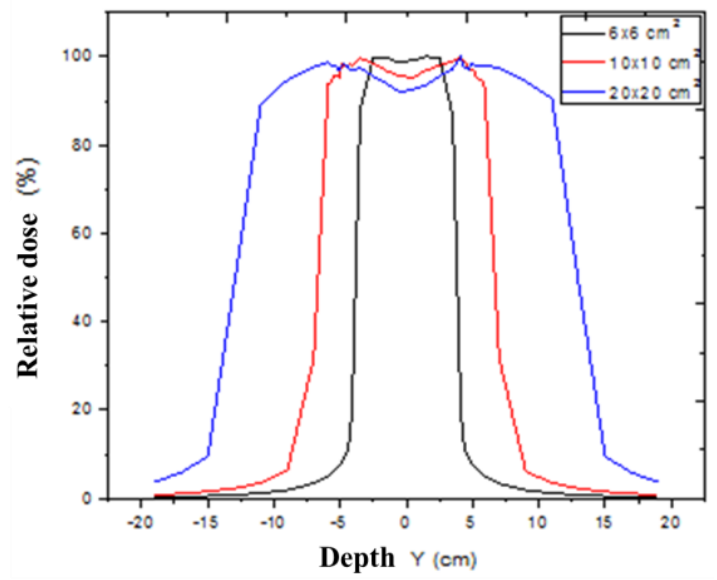

(b)

Figure 5. Variation field size at $6.1 \mathrm{MeV}$ electron energy beginning with SSD $100.1 \mathrm{~cm}$ and depth of $10 \mathrm{~cm}$. (a) PDD graph shows the depth dose distributions (b) The graph shows the dose profile dose distributions to the $\mathrm{Y}$-axis field.

Dmax $100 \%$ by varying the pitch, is at a depth of $1.5 \mathrm{~cm}$ to field size , And the maximum dose of $100 \%$ contained at a depth of $0.6 \mathrm{~cm}$ for field size the larger size of the field size affect the value of the dose in the build-up and fall-off due to the scattering medium which is in line with the increasing size of the field size use. When the size of the field size will be enlarged to affect of increasing value in the dose build-up and dose in the fall-off area. This phenomenon is caused when the size of the field size is enlarged, the contribution of the electrons from the photoelectric effect and scattering radiation is also becoming increasingly, substantial role in causing the dose also increases with depth up to the maximum dose at a certain depth or Dmax.

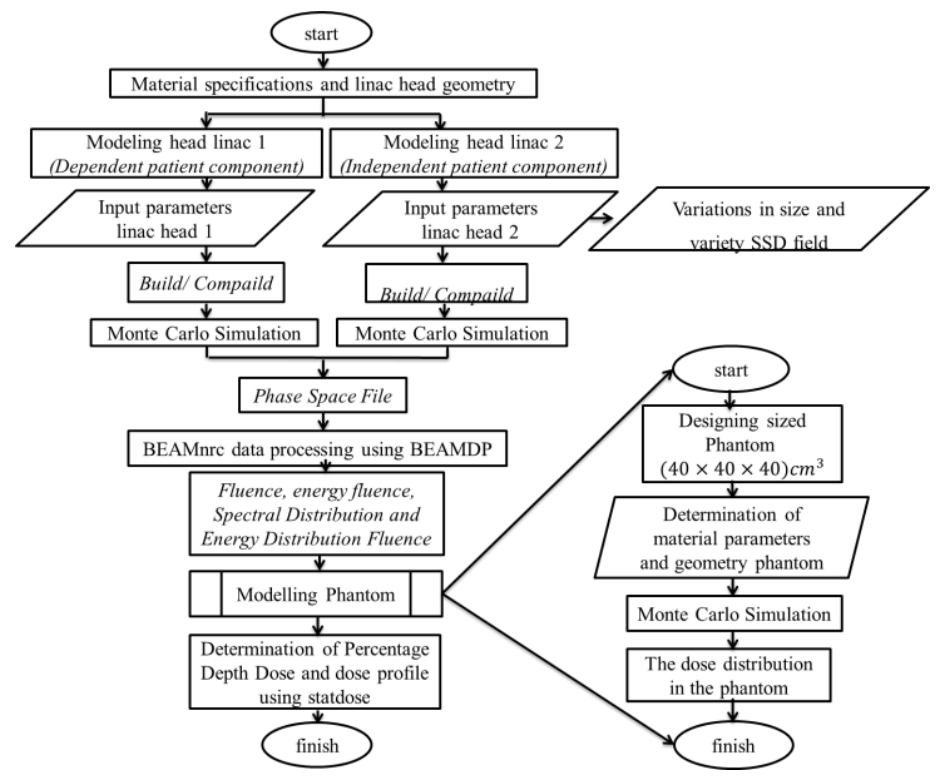

Figure 6. Flowchart Research

Dose distributions will increase along with the larger field size due to the effects of electron more contributions received or absorbed by the material.

\section{CONCLUSION}

Dmax with a dose of $100 \%$ less likely to experience changes in the SSD $80 \mathrm{~cm}, 90 \mathrm{~cm}, 100.1$ $\mathrm{cm}$ at a depth of $0.6 \mathrm{~cm}$ and changes in the SSD 120 $\mathrm{cm}$ with a depth of $1.3 \mathrm{~cm}$. Dmax $100 \%$ by varying the pitch, is at a depth of $1.5 \mathrm{~cm}$ for field size , and the maximum dose of $100 \%$ contained at a depth of $0.6 \mathrm{~cm}$ for field size the larger the field size affect the value of the dose in the build-up and fall-off due to the scattering medium which is in line with the increasing of the field size is used. PDD curve at distance variation is smaller SSD and field size greater then the dose distribution will increase. This phenomenon is caused due to the contribution of electrons results radiation photoelectric effect and scattering is more dominant until maximum depth at certain dose. It began to decline with the deepening of the depth or after a maximum dose depth. Dmax The symmetry of the dose profile curve is very important because of the dose profile curve can be seen from the symmetry of the mean photon beam dose homogeneously dispersed in the medium area. 


\section{ACKNOWLEDGMENT}

The author would like to thank the Department of Physics, Faculty of Science and Technology State Islamic University Sunan Gunung Jati Bandung for its support in this study and Roger Soh School of Physical and Mathematical Sciences, Nanyang Technological University of Singapore on data sheet linac Varian Clinac iX. The research was funded by grants thesis Department of Physics, Faculty of Science and Technology State Islamic University Sunan Gunung Jati Bandung.

\section{REFERENCES}

[1] Wahyuni AR, analysis of the relationship absorbed dose to the distance the radiation source to the surface of the medium (SSD) and wide field illumination of aircraft Linear Accelerator(LINAC), concentration of Medical Physics Department of Physics, Faculty of Mathematics and Natural Sciences, Universitas Hasanuddin, Makassar (2013).
[2] Khan, F. M, The Physics of Radiation Therapy (FourthEdition),William and Wilkins, Baltimor (2010).

[3] Choirul Anam, Monte Carlo Simulation for Electron Contamination At X-ray Image $6 \mathrm{MV}$ linac Aircraft Production ElektaSL15,MIPA UI, Depok (2010).

[4] E. Mainegra-Hing and DWO Rogers I. Kawrakow, EGSnrcMP: the multi-platform environment for EGSnrc: National Research Council of Canada Ottawa, 2006.

[5] Ridwan Ramdani, Comparison of dose distribution VMAT $10 \mathrm{MV}$ photon beam in esophageal cancer using Monte Carlo (MC) and Analytical Anisotropic Algotithm(AAA),Bandung Institute of Technology (2015). 